\title{
How to boost the production of free services: In search of the holy referee grail
}

\author{
Marcel Canoy* Daan in 't Veld ${ }^{\dagger}$
}

May 25, 2014

\begin{abstract}
This paper argues that delivering free services is driven by a combination of motivations, and explains how policy makers or other principals can exploit them to deal with underproduction of free services. We identify intrinsic motivation, respect and gratitude as main examples of underlying motivations. What is essential for free services is that there is considerable heterogeneity in how these motivations are valued across the population. We show how a menu of (monetary and non-monetary) rewards can easily outperform simple compensation approaches since it allows for self-selection and caters for the heterogeneity. As the leading example, we illustrate how a menu can improve the academic referee process. The menu idea can be used in a large set of settings and is potentially very fruitful.
\end{abstract}

JEL classification: D03; L80; I23.

Keywords: free services; underproduction; motivations; menus; referee process.

*TILEC, Department of Economics, Tilburg University and ECORYS, The Netherlands, marcel.canoy@ecorys.com

${ }^{\dagger}$ Corresponding author: CeNDEF, Amsterdam School of Economics, University of Amsterdam, Valckenierstraat 65-67, 1018 XE Amsterdam, The Netherlands, D.intVeld@uva.nl, tel.: +31 20525 4335, fax.: +31 205254349.

The authors would like to thank Johannes Binswanger, Erik Canton and Jan Tuinstra for useful comments. 


\section{Introduction}

As the new Editor-in-chief of the 'Journal of Something in Economics' 1, a relatively young journal that did not yet have the time to develop a strong reputation, one of the authors of this paper wondered what could differentiate the Journal from other Journals. He decided that he would deal with something that had always irritated him as an author: unsmooth referee processes. Could some form of referee reward be introduced? Immediately he bumped into the literature on intrinsic motivation, where it is stated that small rewards would be seen by agents as a 'tip' and would henceforth erode intrinsic motivation (Eisenberger and Armeli, 1997; Fehr and Schmidt, 2004).

The nature of this discontinuity whereby a small change in rewards creates a disproportional behavioral reaction is called 'Payment Discontinuity' in the referee example or 'Type Discontinuity' in other cases such a blood donation (Seabright, 2004). However, for many services it is either commercially unfeasible (referee process) or unethical (organ donation) to have monetary rewards that reflect the true value of the service. Can this problem be solved somehow?

The referee process is an example of a free service: it is delivered (more or less) for free and mainly because of personal motivations. Free services typically suffer from underproduction. For the referee process, this is a result of crowding out of own research and even possible 'punishments' by overenthusiastic editors who overburden good performing referees. The impact of underproduction is serious: referee processes delay progress in entire fields of science.

There are many other examples of free services. Take the classic (Titmuss, 1970) case of blood donation. It is delivered for free, but small payments could undermine the motivation. And here too suboptimal levels are produced. The same applies to organ donation or voluntary work. In all these examples, the suboptimal social levels of these services raise serious concerns. The notorious lack of organ donations has serious ramifications for public health.

This article starts with the question what motivates people who deliver free services. It is doubtful whether the simple principle of 'pay enough or don't pay at all' tells the full story (Seabright, 2004). As noted by Gneezy et al. (2011), what matters in the discussion of incentives is 'the form in which they are given (especially monetary or nonmonetary) [and] how they interact with intrinsic motivations and social motivations'.

We show that the delivery of many free services is driven by a combi-

\footnotetext{
${ }^{1}$ The real name of the Journal is not relevant.
} 
nation of intrinsic motivation and extrinsic motivations such as respect and gratitude. As pointed out by Kreps (1997), such motivations are often viewed collectively as a 'social norm', but distinguishing them will be essential for understanding free services. Furthermore, different people give different weights to each of the motivations. A principal seeking to enhance the production of free services has to work out how these motivations are distributed over the population and what can be done to lift production in a cost-effective way.

As a possible solution we propose that a menu can do the trick for the principal to boost the production of free services. Crucially, a menu deals with the heterogeneity of the population in a cost-effective way, provided that the menu costs are limited. People have different motivations, and it is too costly for the principal to find out which person has which combination of motives or which weights. Instead, the menu lets people choose themselves, a common trick in economics, yet underused in this context. In this way, underproduction is reduced against low costs.

It is surprising that exploiting heterogeneities through menus, which is pretty common in e.g. telecom markets, in marketing or indeed in restaurant menus, seems to be an untrodden path in the world of free services. In economic research, the self-selection mechanism of menus has been presented as a device for indirect price discrimination under incomplete information (Maskin and Rile, 1984). The possibilities of menus to provide for heterogeneity in personal motivations are up till now insufficiently acknowledged. Still it is very relevant, as the menu can be adapted to many free services.

In fact, our theory is also applicable to services that are close to free, in the sense that they are delivered below market price or wage. Think of painters or people working for governments or NGO's. Equally so, the theory is applicable to free services where it is less obvious that they are underproduced. Think of Wikipedia entries or philanthropy in the US. Although those cases may appear perhaps less interesting for a public policy point of view, they can convey useful insights for the services that are underproduced.

We provide a simple model of free services inspired by Bénabou and Tirole (2006). By modeling personal motivations formally, we make clear how the menu affects the agents' decision to deliver free services and under which general conditions the menu is the best option. Our close focus allows us to be straightforward in the reasoning and applications and to enrich the discussion by pointing at solutions that also have practical value to principals facing underproduction of free services.

This article first illustrates in Section 2 what the motivations for delivering free services are by the referee example. Section 3 digs deeper into the nature of intrinsic motivation and other extrinsic motivations. The heterogeneous preferences are modeled in Section 4 to suggest the solution of a menu. 
Section 5 elaborates the menu idea in depth for the example of the referee process. Section 6 concludes.

\section{Explaining the general idea by the referee example}

Unsmooth referee processes have been documented thoroughly; see e.g. Thompson et al. (2010). One reason for this problem is that motivation for delivering the service is complex. While intrinsic motivation is an important driver, various fuzzy extrinsic motivations exist in addition. Different people have different weights for each of these motivations. Due to the complexity, institutions and incentive mechanisms do not sufficiently take into account the various motivations and henceforth fail to produce satisfactory results.

For practical purposes we will concentrate in this paper on two of the fuzzy extrinsic motivations, namely gratitude and respect. Whilst for 'economic' transactions these motivations are clearly relevant this does not exclude that there are many other motivations, such as anger, greed, envy, that can come into play.

\section{Example What drives a referee to produce a good and timely re- port?}

The pro bono refereeing system builds on the gift relationship principle of Akerlof (1982): "I referee because in return someone else referees my papers. Overall I break even." But Leslie (2005) concludes that "Nevertheless, the opportunities for free-riding and lethargy when faced with a specific refereeing task need not be spelt out, much as the public spiritedness behind the present system is commendable. Sometimes refereeing can inform the referee and is willingly done but this is often not the case." What drives this concern? One reason sometimes mentioned (Canton, 2005) is that refereeing is the part of a multitask package of researchers that is least observed and therefore least rewarded. Facing hard incentives of teaching and publishing can erode the motivation to referee.

In reality people are driven by a variety of motivations and constraints, and different people have a different mix of them, also in refereeing. Bob always performs well, since he is curious or considers it his duty. Christine also performs consistently, but she does it because she wants to be liked by editors, she cheekily indulges on the power it gives her over authors and her thesis supervisor seems to find it important. Fred has a mixed performance since he lets the subject determine whether or not he puts in the effort 
('what is in there for me'). Karen does a good job but always too late since she is afraid to be asked too many times by the editors. And then there are combinations of these motivations, there are many other motivations that we have not mentioned, and constraints differ as well. In a simple A-B-C mode it looks like:

A. You referee the paper because you consider this a moral duty related to be a professional scientist [Intrinsic motivation].

B. You referee the paper since it gives you a good feeling to be nice and you receive the gratitude by the author and/or editor [Gratitude].

C. You return it since this improves the respect you obtain from the profession [Respect].

Interestingly, the variety in motivations is missed by most scientific studies on the referee process. Using a survey of economic journal editors, Freedman (2000) finds that the main problem is an inherent lack of accountability and goes as far as saying that self-interest cannot play a role in refereeing; it is vaguely suggested that referees are instead driven by "professional obligation'. The limited theoretical work has focused on the idea that referees are concerned with the quality of the journal (Engers and Gans, 1998), which might lead to feedback loops in quality (Chang and Lai, 2001). Finally, Azar (2008) models referees as influenced by social norm, but again how this norm is shaped by personal motivations is exogenous. It is clear that the simultaneous presence of different underlying motivations has not been acknowledged.

In the referee example there could be even more direct additional motivations than mentioned so far: the hope for a monetary reward, the hope to soften editors or the ability to learn something from the refereed paper in this case. What is crucial is that these direct/financial motivations are not too dominant (otherwise the service becomes 'normal') and intrinsic motivation not too strong (otherwise the service will not be underproduced).

\section{Different motivations}

It has been pointed out before that monetary incentives are not always all that matter. Fehr and Fischbacher (2002) for example argue that so-called 'social preferences' affect outcomes of competition and cooperation by means of simple experiments. However, the literature on motivations is rarely clear what exactly the different motivations are, confusing intrinsic motivation 
with non-monetary or fuzzy extrinsic motivation. In Harsanyi's famous quote "People's behavior can largely be explained in terms of two dominant interests: economic gain and social acceptance" (Harsanyi, 1969), the confusion becomes clear. The problem is that 'social acceptance' is too broad to be useful for policy analysis.

This section digs deeper into the nature of motivations in order to identify the main and economically distinct forms of motivation. As in the example of the referee process, we stick to intrinsic motivation and two forms of extrinsic motivation: respect and gratitude. Each of them is treated separately in the next three subsections, drawing from economic and psychology literature. Other examples will illustrate how these motivations affects the production of free services. ${ }^{2}$

\subsection{Intrinsic motivation}

We explicitly distinguish between intrinsic motivation and non-monetary extrinsic motivation. The distinction is so important since principals who want to influence effort levels have to use very different incentives depending on what drives people. Intrinsic motivation is defined as the sum of social, religious, or moral considerations that cannot be directly boosted by a principal, at least not in the short run. For the principal, it is therefore necessary to focus on different forms of extrinsic motivation which we will cover below. Intrinsic motivation can be influenced in the longer run if social, religious or moral norms change.

There is one way though in which intrinsic motivation can be negatively influenced in the short term, and this is the well-known crowding-out effect of monetary incentives. The reason is not that the norm changes, but by inclusion of monetary incentives changes the nature of the relation between the individual and the principal. Take the case of a blood donor. If the donors act voluntary and no monetary rewards are included the principal signals that it relies on the non-monetary motivations of the individuals (intrinsic and extrinsic). Including monetary incentives changes the nature of the service and the relationship between principal and donor, with the possible consequence that the individual does not feel inclined to deliver anymore. There are many studies that show this point as for example Lacetera and Macis

\footnotetext{
${ }^{2}$ Taking a historical perspective, Bruni and Sugden (2007) discusses how neoclassical economics has been pursuing to develop a science completely independent of psychology. A fundamental problem of this pursuit is to determine the borders of the domain of economics. From this point of view we argue that free services fall outside the domain of neoclassical economics with an exclusive focus on material self-interest.
} 
$(2010) .^{3}$

Kreps (1997) states that there are different reasons for people to follow social norms. Adherence to norms can be (i) outright personally beneficial or, despite being costly, it can be (ii) overall beneficial within a society or (iii) personally desirable per se. Following Kreps (1997), we disregard the first explanation as being uninteresting, but focus on the difference between the last two. Explanation (iii) falls under our definition of intrinsic motivation, and we will call (ii) non-monetary extrinsic motivation. It becomes clear that intrinsic motivation can only change if the norms themselves change whereas in the case of non-monetary extrinsic motivation, the parameters can change without the norms having to change. Notice that the desirability per se can have different underlying motives itself, e.g. it can be based on reciprocity, sheer kindness or fundamental religious beliefs, but this subdivision is not what interests us in this paper.

Linking motives to the psychological literature, let us look at the multifaceted nature of motivation as developed by Reiss (2004). The author claims that he can make a complete list of motives of human behavior (Power, Curiosity, Independence, Status, Social contact, Vengeance, Honor, Idealism, Physical exercise, Romance, Family, Order, Eating, Acceptance, Tranquility, Saving). Some of these are not changeable in the short term and fall within our notion of intrinsic motivation for free services, namely Curiosity and Idealism.

When we look at the remaining categories, it becomes obvious that many categories from the list will rarely be relevant in economic transactions. We will select and translate respect (Status, Social contact) and gratitude (Honor, i.e. the tendency to obey a moral code) as the main forms of nonmonetary extrinsic motivation. These will be explained further in the next two subsections. It is not to say that the other categories are never relevant but our idea is best illustrated by taking a limited number of (clearly relevant) motivations. The same idea can easily be extended taking other motivations into account.

The problem with the (economic) literature is that it often assumes a 0-1 case: either monetary incentives crowd out intrinsic motivation or it does not; either people are intrinsically motivated or they are not. Kreps (1997) shows that such 0-1 thinking does not do justice to the complexities of motivations. This paper therefore builds on Kreps (1997) and Reiss (2004) and provides a richer set of motivations.

\footnotetext{
${ }^{3}$ Lacetera and Macis (2010) state that the particular form of the monetary incentive is important: there is an additional aversion to receive cash. Our idea can be interpreted as a new form of offering a monetary incentive, namely through a menu with alternative non-monetary options.
} 


\subsection{Respect}

A person may buy services given his budget constraint, but sometimes he is helped by others for different reasons. How do people know this person is the one to help? In this context, respect serves as a signaling device for abilities that are difficult to observe. In a formal model, Rege (2008) describes social status when people engage in different types of interactions. The working of respect is particularly well explained in an internet essay by Johnson (1999):

"Anthropologists have observed respect working in the real world [...]. In hunter-gatherer societies even the best hunters will only bring meat back occasionally. When they do they usually have far more than they can cope with at once. The obvious solution is to share. But this brings a problem: what about the lazy hunter who never brings anything back but still gets a share of everyone else's hard work?

Respect is the solution to this problem. Every time a hunter brings home the bacon he goes up in the estimation of the rest of the tribe. This translates into choicer cuts of all the meat, not just his own. In effect the hunter has traded meat today for meat tomorrow, using the respect of others as a store of value on which to draw during lean times."

In the quotation above, Johnson observes that respect can solve the freerider's problem in societies when direct rewards are insufficient. The important difference with money is that respect cannot be traded. This also explains why sometimes money can reduce respect. Quoting Johnson again:

This limitation means that money has a divisive effect on gift cultures. A member of a gift culture is tied to that culture: the store of respect he has earned is in the heads of other members of the culture and cannot be transferred elsewhere. But if the same individual obtains money instead of respect then that money can be taken elsewhere, and even traded for respect in another gift culture if desired.

In the economic literature, respect is shown to have far-reaching effects in growth models, tax policy, and the distribution of income (Rege, 2008). The theory is equally relevant for the provision of underprovided free services. If a solution contributes to the respect of potential 'producers' of the free service, the number of effective producers will increase. One should only think of the success of Wikipedia to understand how this works. Since there are likely network effects associated with respect (club feelings) any solution that will improve respect is likely to enjoy scale economics. Notice also that unlike intrinsic motivation which is (certainly in the short run) 'given', respect can easily be influenced by the principal. 


\subsection{Gratitude}

There is another relevant strand in the literature that deals with motivations for providing gifts and the psychology of gratitude (Amegashie, 2006; Andreoni, 1990; Brekke et al., 2003; McCullough et al., 2001). From McCullough et al. (2001) we infer that gratitude can be defined by three moral functions:

"[G]ratitude is conceptualized as a moral affect that is analogous to other moral emotions such as empathy and guilt. Gratitude has 3 functions that can be conceptualized as morally relevant: (a) a moral barometer function (i.e., it is a response to the perception that one has been the beneficiary of another person's moral actions); (b) a moral motive function (i.e., it motivates the grateful person to behave prosocially toward the benefactor and other people); and (c) a moral reinforcer function (i.e., when expressed, it encourages benefactors to behave morally in the future). The personality and social factors that are associated with gratitude are also consistent with a conceptualization of gratitude as an affect that is relevant to people's cognitions and behaviors in the moral domain."

Gratitude is different from intrinsic motivation or respect. Take e.g. somebody who is prepared to work for an NGO for a below market wage. The individual may be intrinsically motivated, independently from other people. Alternatively, he or she could desire respect from peers, friends or relatives. But now suppose that the individual senses the gratitude of people that directly benefit from the work of the NGO. They he or she could accept a smaller salary even in absence of any intrinsic motivation or any respect argument.

The gift and gratitude effects have two layers. People are inclined to give, i.e. to deliver a free service in part of the gratitude they receive as a consequence of delivering. If the principal provides a non-monetary reward in return an added effect can occur, increasing the motivation of gratitude. Even though the the second layer exists, gratitude is different from standard reciprocity arguments (Fehr and Schmidt, 2004). Gratitude can be shown with the first layer only, and moreover the size of the gifts in the second layer is not too relevant.

The theory of gratitude is obviously of importance to motivate possible solutions for the suboptimal levels of free services. In particular if the solutions can work on (b) and (c) functions of gratitude in the quotation above, it would stimulate actions in a positive direction.

To summarize, the literature on intrinsic motivation, respect and gratitude shows what is behind the different motivations in delivering free services, but there are not many attempts to combine the insights from the different 
strands. Our paper does precisely this, and applies the theory to free services such as the referee problem.

\section{A simple model}

In this section we formalize the idea that people may have different motivations to supply free services, and that a menu can be the best solution. We develop a principal-agent model inspired by Bénabou and Tirole (2006), but much simpler and smaller in scope. They investigate the effects of rewards on signals people send when performing good deeds. We drop the uncertainty of others about an individual's motivations and the resulting signaling game. The key idea here will be that a menu can support the different motivations and solve the crowding out of free services. The main outcome of the model will be the optimal number of options in the menu.

We first consider the individual problem for an agent who has to decide whether or not to supply a free service, denoted as $a_{i} \in\{0,1\} .{ }^{4}$ Although the activity is costly for him (bearing utility costs $c_{i}$ ), there are motivations in various dimensions. Assume every agent $i$ has as personal characteristics intrinsic motivation $\iota_{i}$ and a $n$-vector of relevant reward valuations $e_{i}$, representing different forms of extrinsic motivation. In particular, these include the individual valuation of money, respect from society and gratitude.

Under the absence of rewards, each agent will provide the service if the intrinsic motivation outweighs the utility costs of supply, or

$$
a_{i}=I\left(\iota_{i}>c_{i}\right)
$$

where $I$ denotes the indicator function. For many agents, however, a motivation boost is needed to overcome the threshold $c_{i}$. To keep things simple, we will assume that intrinsic motivation can be replaced by some form of extrinsic motivation if it is triggered by the reward related to this extrinsic motivation. Let us order $e_{i}$ such that $e_{1, i}$ is the valuation of money. When facing a monetary reward, for example, the individual will simply evaluate the trade-off $e_{1, i}$ versus $c_{i} .{ }^{5}$ Similarly, the valuations in $e_{2, i}, e_{3, i}, \ldots, e_{n, i}$ can be triggered by non-monetary rewards and will be compared with $c_{i}$. Think of creating high visibility of the action to society that increases respect, and ways in which the principal can express gratitude.

\footnotetext{
${ }^{4}$ Bénabou and Tirole (2006) allow for continuous participation levels, covering the intensity of participation. Our model can easily be generalized in this direction.

${ }^{5}$ Of course, the reward may interact with intrinsic motivation, so that the tradeoff becomes $\iota_{i}+e_{1, i}$ versus $c_{i}$ as in Bénabou and Tirole (2006). The effect of this simplification will be discussed below.
} 
Note that retaining the same threshold $c_{i}$ over all motivations assures that we are comparing motivations that will separately induce the same production level. This goes without loss of generality, because the individual valuation of money can be scaled to the (fixed) amount of the monetary reward. We are interested in how the motivations, heterogeneously spread over the population, will interact by using a menu.

Now consider the principal who is concerned with the participation level. The principal has to make some beliefs about the distribution of characteristics among the agents, $f\left(v_{i}\right)$, where $v_{i}=\left(\begin{array}{c}\iota_{i} \\ e_{i}\end{array}\right)$ on some domain $D \subset \mathbb{R}^{n+1}$. Taking into account the rewarding costs $r_{i}$ per agent and fixed costs $F C$, the welfare function of the principal is:

$$
W=\int_{D}\left(a_{i}-r_{i}\right) f\left(v_{i}\right) d v_{i}-F C
$$

The question arises how the principal should set the rewards to maximize his welfare. Offering a menu provides an extra decision for the agents. Because of the self-selection, every agent $i$ chooses the reward that gives the highest of the contributions in $e_{i}$, or sticks to its intrinsic motivation $\iota_{i}$. The agent's action now depends on the maximum valuation that can be reached within the menu ${ }^{6}$ :

$$
a_{i}=I\left(\max \left(v_{i}\right)>c_{i}\right)
$$

So far the model has provided a fairly general, though stylized description to any problem of free services. Now we want to find its outcome in an illustrative case. The following assumptions are made to solve the model:

1. The valuations $v_{i}$ are drawn from a multivariate uniform distribution over $D=[0,1]^{n+1}$. We fix $0<c<1$ for all $i$, so that only a fraction of the agents will participate. Note that we assume that the individual characteristics are independent from each other. While for some people motivations may be correlated, assuming independence is reasonable at the population level.

\footnotetext{
${ }^{6}$ This expression makes clear that adding extra options always favors the agents. Verme (2008) discusses alternative views of the freedom of choice that allow utility to be possibly decreasing in the size of the choice set. Ignoring this possibility slightly overestimates the optimal number of options.
} 
2. There are fixed costs $k$ for every of the $n$ options included in the menu, so $F C=n k$. All rewards bear cost $r$ to the principal per individual. It is required that $r<c$. If rewards were more costly to the principal than providing the good is for the agents, it would not be optimal to offer rewards. It will be convenient to define the effective costs per option $K \equiv \frac{k}{1-r}$. These are the fixed costs taking the individual rewarding costs $r$ into account.

The assumption of equal costs eases the exposition. In practice, it will not be often satisfied and the principal will order his options $K_{1}<$ $K_{2}<\ldots<K_{n}$ and choose the first options from this list.

3. Intrinsic motivation is crowded out if only a monetary reward is offered, but not if a menu is offered containing a monetary reward. So if and only if a single monetary reward is offered, equation (3) will be changed from $a_{i}=I\left(\max \left(\begin{array}{c}\iota_{i} \\ e_{1, i}\end{array}\right)>c\right)$ to $a_{i}=I\left(e_{1, i}>c\right)$.

Straight-forward calculations show that under these conditions the welfare function equals:

$$
W=\left\{\begin{array}{cl}
1-c & \text { if no rewards } \\
(1-c)(1-r)-k & \text { if only a monetary reward } \\
\left(1-c^{2}\right)(1-r)-k & \text { if only a non-monetary reward } \\
\left(1-c^{n+1}\right)(1-r)-n k & \text { if a menu with } n \text { rewards }
\end{array}\right.
$$

The driving force of the menu with $n$ options is that the fraction of nonproducing agents $c<1$ decreases to $c^{n+1}$. Of course, this comes at a cost, depending on $r$ and $k$.

It follows directly from the assumptions that offering only a monetary reward decreases the welfare of the principal. While there are extra costs for rewarding, the number of participating agents remains equal, as intrinsic motivation is eroded by the monetary motivation and both are identically distributed. Offering a single non-monetary reward is therefore given our assumptions always a better possibility than a single monetary one.

A menu with multiple options is often even better than a single reward, because by self-selection of the optimal reward most agents are induced to participate. The menu can be solved for the optimal number of options. The optimization problem can be written as

$$
\max _{n}\left(1-c^{n+1}\right)-n K
$$


and gives

$$
n^{*}=\frac{\log \left(\frac{K}{-\log (c) c}\right)}{\log (c)}
$$

The partial derivative $\frac{\partial n^{*}}{\partial K}=\frac{1}{K \log (c)}$ indicates that the optimal number of options $n^{*}$ decreases in $K(\log (c)<0$ for $0<c<1)$. The relation with agents' production costs $c$ is non-monotone as will be discussed below.

As a corollary of this result, we can find when principals should decide not to have any reward at all. From $n^{*}>0$, it follows that

$$
K<-\log (c) c
$$

is a necessary condition for having at least one reward. ${ }^{7}$ This condition is restrictive if $c$ is close to either 0 or 1 , as $\lim _{c \rightarrow 0}[-\log (c) c]=\lim _{c \rightarrow 1}[-\log (c) c]=$ 0 . For intermediate $c$, offering a reward is effective for relatively high $K$. The interpretation is relatively straightforward. For low $c$, agents face low costs of engaging in the desired activity so that a reward yields relatively low extra value. Since the reward is costly in itself, the reward does not outperform straightforward ways of production for low $c$ 's. If $c$ increases, the merits of the reward come into play. If $c$ increases further, it becomes increasingly hard for the reward to outweigh the costs, as few extra agents are induced to production.

Figure 1 presents the optimal number of options (plotted as a real variable) depending on $K$ and $c$, showing how this number ranges between 0 and 8 for a wide range of parameters. For very small $K$, the menu can be made arbitrarily large. Notice that for a given $K$ (say $K=0.1$ in Figure 1 ), the optimal number of options is highest again for intermediary values of $c$. A similar intuition as for the first reward applies: in the intermediary range of $c$ for every $K$, an additional reward has the highest merits, so there $n^{*}$ will be the highest.

There are two simplifications in the analysis above that might bias the size of the menu found to be optimal. First, there might be some options that have lower effective costs $K$, so menus could be extended by including cheap options. Second, we have simplified the maximization problem so that intrinsic motivation was not added to the motivation triggered by the reward. This last simplification put upward pressure on the size of the menu.

\footnotetext{
${ }^{7}$ If options have different effective costs $K$, the condition applies to options with the lowest costs $K_{1}$.
} 


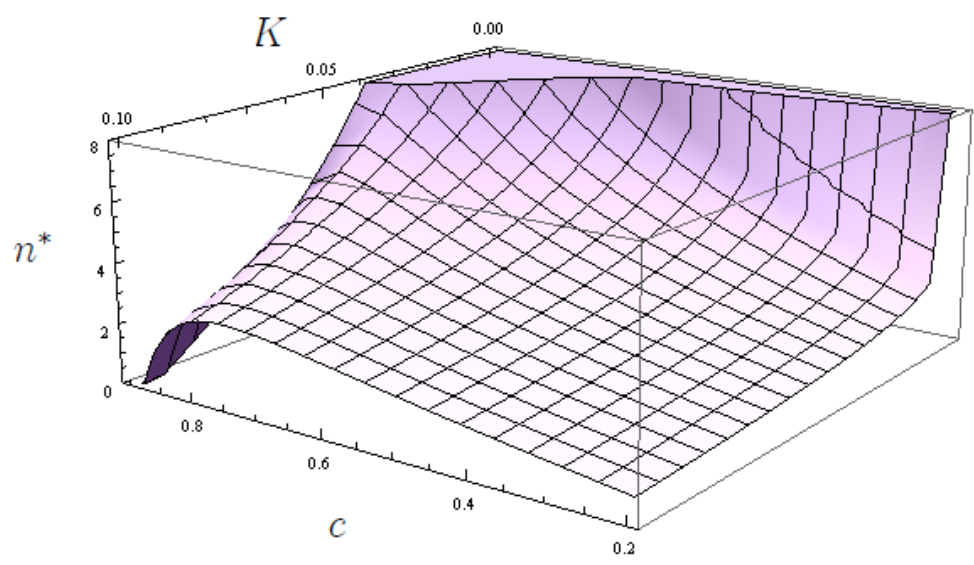

Figure 1: The optimal number of options in the menu $n^{*}$.

\section{Applying the menu to the referee problem}

With the simple model in the back of our heads, we will now elaborate the example of the referee process. Consider the following hypothetical letter to referees and observe how the menu caters for different forms of respect, gratitude and intrinsic motivation:

The Journal wants to guarantee a smooth referee process. Since we fully realize that being a referee is not always a thankful job, we can offer the following variable (modest) referee rewards:

A. We will not ask you again as a referee for the next year. [Respect]

B. Your name will be published in a list of good performing referees, both in the Journal as on our website. [Respect]

C. The publisher will donate 50 euro to a charity fund. [Gratitude]

D. The publisher offers you 50 euro. [Extrinsic monetary motivation]

Please indicate your choice out of the four reward options. It goes without saying that the reward is only awarded if the referee is on time with a serious report.

Let us look at the logic behind this letter and the menu in detail. First of all, the opening statement in the letter signals that the editors show respect for referees, enhancing the possibility that the referee accepts the job. Option $\mathrm{A}$ is included as another important signaling device. Of course those referees 
that are afraid to be overburdened can choose for this one, but it is important even for the referees that do not choose this option that the Journal credibly signals that it takes time of referees seriously. This could be seen as an external effect: the fact that other referees can choose this option adds to respect.

Option B is a variant of option $\mathrm{A}$. It adds to the respect factor, but while option A caters for personal respect, option B goes for public respect, both forms being potentially relevant. In particular referees in the start of their career can choose this option. Note that option B has considerable fixed costs of producing and distributing the list, while the variable costs of adding another referee to the list are low. These costs generate a trade-off between menus with or without this option, or publishing the list as an extra reward outside of the menu, as is now already done by some journals.

Option $\mathrm{C}$ adds to the gratitude feeling. Importantly, issues of intrinsic motivation erosion are out, since referees who do not wish to receive anything can go for options A, B or C. Assumption 3 above, or perhaps a less extreme version, is clearly justified in this context.

Option D is the standard commercial option, and takes care of referees who are not too sensitive for gratitude or respect arguments and do not suffer from erosion of intrinsic motivation. Notice that there are many variants of this in the referee 'market': a fixed amount of money, free subscriptions, et cetera.

The idea of the menu is that it can increase the probability of accepting the job and delivering on time while the extra costs to the principal remain in check. The menu both caters for heterogeneity and possible uncertainty through self-selection. In addition, the menu has hidden benefits as well. The respect factors also show up (but possibly not to the same extent) even if the participants do not choose for the option that influences respect. The reason is that the menu generates network externalities. If the menu increases the number of people delivering the service, the total respect of delivering the service goes up. As long as the menu is sufficiently smartly designed so that respect options are indeed chosen by people, the network externalities will ensue.

As mentioned above, the menu can be extended to cater for different variants or motivations, but the risk of a Christmas tree of variants is that it becomes fuzzy, thereby less catchy and potentially eroding motivation. Instead, our menu as a total caters parsimoniously for the variety of motivations such as gratitude and respect. In this sense it outperforms other menus that only support the monetary (or materialistic) motivation, such as the one offered by the Journal of Economics Dynamics and Control:

- a one-year free electronic subscription to the JEDC, or 
- a submission fee waiver for a JEDC submission, or

- a US\$ 100,- transfer into your Paypal account, or

- $\quad a € 70$,- transfer into your Paypal account.

Other journals don't do much better. The AER just pays $\$ 100$ for timely reports. Econometrica bets on intrinsic motivation plus a glimpse of respect: "The majority of the work at the journal, of course, is done by the many anonymous referees who provide timely and thorough reviews. We thank them for their generous efforts" and then they list the referees. The Journal of Economics just includes a list with do's and don'ts. The Journal of Public Economics does not provide rewards but states that "Most reviewers are authors, researchers, or sometimes editors in their own right. The peer review process allows these authors and editors an opportunity to use and develop their own expertise in a number of significant ways." ${ }^{8}$ We do not want to talk down to these Journals. The point is that no journal seems to get it right and that much can be improved in the referee process in general.

Let us now push the analysis one level further by exploiting the results from the model. Given the result that a menu is the preferable modus of generating welfare gains in the delivery of free services if the participation gains outweigh the costs, it is instructive to verify on the basis of the referee example how conditions can be influenced such that those welfare gains can indeed be realized.

First of all, we propose that one of the bigger Journals could run an experiment where part of the referees would receive a menu similar to the one we describe above. To control for the heterogeneity in the referees, a control group should be assigned of referees that for example simply receive a fixed amount of money. The benefits of the menu are likely to outweigh the costs, as long as different motivations are catered for intelligently.

Participation levels can obviously be further increased if the menu options are tailored to observed motivations. To find out more about relevant motivations, Journals can do surveys among referees. Journals can then choose a limited number of options that matter most and gear the options towards those. The number of options will naturally depend negatively on the fixed and marginal costs of the rewards, but most importantly, they should genuinely different motivations to be effective. Subsequently, they can do randomized trials to see which menu options perform best for those motivations. When you see the list of the Journal of Economics Dynamics and Control mentioned above (and there are many similar ones) it becomes clear that motivations such as respect and gratitude have not been taken into account. These menus simply miss the clue completely, as they focus only

\footnotetext{
${ }^{8}$ All information about referee rewards was taken from publicly available web pages.
} 
on the monetary incentive.

In case respect is an important motivation, participation levels can also be increased by using a public leverage. If respect can be granted not only in a personal way but also in a public way, the gains from respect will increase for the participant, and hence the probability of participation. The internet provides ample opportunities to find creative options to boost participation levels without raising costs too much. This includes various leveraging possibilities. In particular a bigger Journal can create mass with this, so that leverage can be reached by involving the most popular charity funds, who can use this action to their benefit by giving it forms of publicity. This can then benefit participants, by pushing on the respect button. Such forms of mutual benefits are rife on the internet and social media.

There are also ways to reduce costs. In case of a monetary reward option the price elasticity can be calculated, avoiding underpayments or excessive payments. Costs can further be reduced by creating (non-monetary) options with zero or low marginal costs. Option B which offers to publish names in a list of good performing referees is a good example of this. Furthermore, in the option $\mathrm{C}$ it is not obvious that a monetary payment needs to be paid to a charity fund. Creative forms can be created in the form of a 'friends of' community for a certain charity fund.

Taken together, it is clear that a well-designed reward menu with heterogeneous options can improve the referee process. The effects are likely to be both in the quantity, the quality and the timing of referee reports. We showed that there is a large potential of yet unused options to boost the process of refereeing, thereby realizing welfare gains. Given the fact that the referee process is a crucial input of the scientific production, this seems a missed opportunity.

\section{Conclusion}

This paper argued that delivering free services is driven by a combination of personal motivations. As main motivations the paper used intrinsic motivation, respect and gratitude. As the leading example of a free service we used the referee process. The ideas of the paper can easily be applied using other types of motivations and free services.

The extent to which different motivations drive the delivery of free services depends on the type of service delivered, but for any given service there are also (possibly substantial) differences across the population. In the context of the referee process, it is clear that journals and publishers do not exploit the heterogeneity of motivations at all. In all likelihood the referee process 
can be improved substantially without much effort or costs after the different motivations have been identified.

Understanding the motivations of citizens allows principals to design a mechanism that will boost production of free services in a cost-effective way. We illustrated how a menu of non-monetary rewards (possibly with a monetary option included) can easily outperform simple approaches since it allows for self-selection and caters for the heterogeneity. The menu idea can be used in a large set of policy settings (microcredits, organ donation, blood donation, etc.) and is ideally suited for natural experiments. The idea is potentially very fruitful because it is relatively easy to implement, as the case of the referee process illustrates.

\section{References}

Akerlof, G., 1982. Labor contracts as partial gift exchange. Quarterly Journal of Economics 97 (4), 543-569.

Amegashie, J. A., 2006. Economics, gratitude, and warm glow. University of Guelph Working Paper.

Andreoni, J., 1990. Impure altruism and donations to public goods: A theory of warm-glow. Economic Journal 100, 464-477.

Azar, O. H., 2008. Evolution of social norms with heterogeneous preferences: A general model and an application to the academic review process. Journal of Economic Behavior \& Organization 65, 420-435.

Bénabou, R., Tirole, J., 2006. Incentives and prosocial behavior. American Economic Review 96 (5), 1652-1678.

Brekke, K., Kverndokk, S., Nyborg, K., 2003. An economic model of moral motivation. Journal of Public Economics 87, 1967-1983.

Bruni, L., Sugden, R., 2007. The road not taken: How psychology was removed from economics, and how it might be brought back. The Economic Journal 117, 146-173.

Canton, E., 2005. Power of incentives in public organizations when employees are intrinsically motivated. Journal of Institutional and Theoretical Economics 161, 664-680.

Chang, J., Lai, C., 2001. Is it worthwhile to pay referees? Southern Economic Journal 68, 457-463. 
Eisenberger, R., Armeli, S., 1997. Can salient reward increase creative performance without reducing intrinsic creative interest? Journal of Personality and Social Psychology 72, 652-663.

Engers, M., Gans, J., 1998. Why referees are not paid (enough). American Economic Review 88, 1341-1349.

Fehr, E., Fischbacher, U., 2002. Why social preferences matter - The impact of non-selfish motives on competition, cooperation and incentives. The Economic Journal 112, C1-C33.

Fehr, E., Schmidt, K. M., 2004. Fairness and incentives in a multi-task principal-agent model. Scandinavian Journal of Economics 106, 453-47.

Freedman, C., 2000. Do economic journals obey economic prescriptions? Review of Industrial Organization 17, 371-384.

Gneezy, U., Meier, S., Rey-Biel, P., 2011. When and why incentives (don't) work to modify behavior. Journal of Economic Perspectives 25, 191-210.

Harsanyi, J., 1969. Rational-choice models of political behavior vs. functionalist and conformist theories. World Politics 21, 513-538.

Johnson, P., 1999. www.sustainablesoftware.info/jahia/Jahia/site/collaborativesource/cache/offonce/pid/421.

Kreps, D. M., 1997. Intrinsic motivation and extrinsic incentives. American Economic Review 87, 359-364.

Lacetera, N., Macis, M., 2010. Do all material incentives for pro-social activities backfire? The response to cash and non-cash incentives for blood donations. Journal of Economic Psychology 31, 738-748.

Leslie, D., 2005. Are delays in academic publishing necessary? American Economic Review 95, 407-413.

Maskin, E., Rile, J., 1984. Monopoly with incomplete information. RAND Journal of Economics 15 (2), 171-196.

McCullough, M. E., Kilpatrick, S. D., Emmons, R. A., Larson, D. B., 2001. Is gratitude a moral affect? Psychological Bulletin 127, 249-266.

Rege, M., 2008. Why do people care about social status? Journal of Economic Behavior and Organization 66 (2), 233-242. 
Reiss, S., 2004. Multifaceted nature of intrinsic motivation: The theory of 16 basic desires. Review of General Psychology 8, 179-193.

Seabright, P., 2004. Continuous preferences can cause discontinuous choices: An application to the impact of incentives on altruism. Institut d'Economie Industrielle Working Paper 257.

Thompson, G., Aradhyula, S., Frisvold, G., Tronstad, R., 2010. Does paying referees expedite reviews? Results of a natural experiment. Southern Economic Journal 76 (3).

Titmuss, R. M., 1970. The Gift Relationship. London: Allen and Unwin.

Verme, P., 2008. Happiness, freedom and control. Journal of Economic Behavior and Organization 71 (2), 146-161. 